\title{
Effect of training on health outcome including fatigue, depression and quality of life in patients with systemic lupus erythromatosus
}

\author{
Manal K. Youssef* (1)
}

\begin{abstract}
Background: Systemic lupus erythematosus is an inflammatory condition that causes significant tissue damage and inflammation, and is more common in women. Despite the fact that medical treatment seeks to prevent flare-ups and organ damage, up to $50 \%$ of patients believe their wellbeing is suboptimal due to unmet needs like exhaustion and pain. The aim of the new systemic lupus erythematosus treatment guidelines is to improve people's quality of life. In people with systemic lupus erythematosus, fatigue is associated with reduced health, exercise capacity, muscle strength, and impairment. The aim of the study is to compare the effects of strengthening exercise on fatigue, depression, and quality of life in patients with systemic lupus erythematosus compared to the control sedentary group. This study included 20 patients with systemic lupus erythematosus from the department of internal medicine, compared to 25 control patients. Both groups had been treated with strengthening exercises for three months. Self-rating depression scale, quality of life questioner, fatigue severity scale, 6 min' walk test, 2 min step test and body mass index (BMI). All parameters were measured before and after 3 months of treatment.

Results: Analysis of data showed that systemic lupus erythematosus has more depression and fatigue than sedentary control. After exercises, both groups have a significant difference between pre and post treatment, but still have a difference between both groups. Both groups have a significant difference between pre and post treatment in 6-min walk test, 2-min step test, and quality of life measured by SF-36. No significant difference was found between both groups pre and post treatment in BMl, although each group has a significant difference between pre and post treatment BMI.
\end{abstract}

Conclusions: Exercises are very important in the management of systemic lupus erythematosus. They help to decrease fatigue, depression, and improve quality of life.

Keywords: Systemic lupus erythromatosus, Exercise, Physical performance, Quality of life

\section{Background}

Systemic lupus erythematosus (SLE) is a condition that primarily affects young people between the ages of 15 and 45 , primarily young women. SLE affects $10-15$ times more women than men [1]. The prevalence ranges from 20 to 150 cases per 100,000 people, with Brazil having the highest rate, and it looks to be rising as the

\footnotetext{
*Correspondence: Manal.kamel@cu.edu.eg; Manal.kamel@hotmail.com Department of Internal Medicine, Cairo University Hospitals, El Saray Street, Giza 12613, Egypt
}

disease becomes more widely identified and survival rates improve. In the United States, people of African, Hispanic, or Asian ancestry have a higher frequency of SLE and more organ involvement than people of other racial or ethnic groups. The 10 -year survival rate is around $70 \%$ [2].

SLE has varying degrees of severity and affects practically every organ system. Periods of flare may alternate with periods of remission over the course of the disease, resulting in disease and therapy-related damage. Lupus flares can range from minor to life-threatening. Most 
patients experience flares of disease activity, followed by periods of remission when their symptoms are relatively quiet [3].

Because SLE causes long-term difficulties, SLE patients are more likely to be sedentary. Reduced exercise capacity due to a change in lifestyle and decreased physical activity. Therapeutic exercise aims to alleviate a number of clinical symptoms in SLE patients by changing the disease's natural course and decreasing the inflammatory process. The most prevalent signs of a long illness were joint deformity, muscle weakness, and deconditioning. Fatigue is another symptom of SLE that causes people to cut back on their daily activities or stop exercising altogether, exacerbating their symptoms. Fatigue is most likely caused by a combination of factors. Cytokines linked to active inflammation, sleep disturbances, a sedentary lifestyle, anemia, depression, stress, and drugs like steroids and beta-blockers are all possible causes. This decline in physical fitness resulted in decreased functional capacity $[4,5]$.

Although they are younger and more likely to be working, tiredness in SLE is related to lower fitness levels, poorer exercise capability, reduced muscle strength, and greater impairment when compared to inactive controls [6]. In this context, exercise training has been used as a non-pharmacological method to help SLE patients with a variety of clinical symptoms.

Nonsteroidal anti-inflammatory medications, antimalarial medicines, glucocorticoids, and immunosuppressive medicines are used to treat SLE patients. The severity of the disease and the function of the implicated organ have a big role in drug selection. However, long-term use of glucocorticoids and immunosuppressive medications can cause bone and muscle atrophy as well as cardiovascular failure [7]. Avascular necrosis, diabetes mellitus, hypertension, and stroke are all risk factors, especially in physically sedentary individuals who spend a lot of time in bed [8].

The goal of this study is to see how strengthening activities affect fatigue, depression, and quality of life in SLE patients compared to a sedentary control group.

\section{Methods}

The study included 20 SLE from the department of internal medicine and 25 sedentary control participants. All procedures were carried out in compliance with the relevant committee on human experimentation's (institutional and national) ethical committee of the faculty of physical therapy, as well as the Helsinki declaration of 1975 , as revised in 2000. All patients who agreed to participate in the study signed informed consent.

Inclusion criteria:
- The subject must be from 25 to 40 years old.

- In the last six months, they've lived a sedentary lifestyle.

Exclusion Criteria:

Renal failure has been present for a long time.

Previous stroke.

Diabetes mellitus.

Heart problems.

Osteoarthritis.

Measurements The following parameters were measured at the beginning of treatment and after three months of treatment.

1- BMI

2- Quality of life (QOL) measured by (SF-36)

3. Fatigue severity score (FSS)

4. Self-rating depression scale (SDS)

5. A 6-min walk test and a 2-min step test were used to assess exercise capacity.

\subsection{Intervention}

The intervention consists of an exercise regimen limited to isolated upper and lower extremity joint ranges of motion as well as limb movement patterns for both groups. It was important to include multiple rest periods to avoid affecting cardiovascular fitness. The workouts were performed three times a week for 50 min each time. The program lasted for three months.

The range of motion exercise also includes muscle strengthening. Stretching activities were followed by isometric and progressive resistive workouts, and the program ended with gentle stretching.

A typical strength program consists of 2 to 3 groups of exercises. Each group consists of 10 repeated isotonic contractions per muscle group, with weights ranging from 1 to 2 pounds depending on the subject's tolerance.

\subsection{Statistical analysis}

Data was statistically described in terms of mean $\pm S D$; the collected data was fed into the computer for statistical analysis and the statistical significance at a confidence level of $95 \%$.

All statistical calculations were carried out using computer programs, Microsoft Excel 2010 (Microsoft Corporation, New York, USA) and Minitab version 19.

\section{Results}

A paired $t$-test was used to compare between pretreatment and post treatment values of the health outcomes, exercise capacity and life quality of each group, and an independent $\mathrm{t}$-test was used to compare between both 
groups. The significance level was set at $P \leq 0.05$ for all tests.

Due to inactivity, SLE causes a decline in aerobic capacity, which leads to obesity. $\mathrm{BMI}=34.8$ before exercise training in sedentary control, which has a lower aerobic capacity than healthy subjects due to fat and inactivity, versus BMI of 35.1 in SLE. Before therapy, no significant difference was found in BMI between both groups $(P=0.292)$ and after therapy $(P=0.606)$. There was no significant difference between the two groups, though there was a significant difference between pre and post treatment in each group $(p<0.001)$.

There are significant differences between the pre and post treatment depression scale, fatigue severity scale, 6 min walk test, 2 min step test, and SF-36 for the sedentary control group, $p=0.001$. There are highly significant differences between pretreatment and post treatment depression scales, fatigue severity scales, 6-min walk test, 2-min step test, and SF-36 in the SLE group, $p=0.001$ (Figs. 1, 2, 3, 4 and Tables 1, 2, 3).

\section{Discussion}

This study was conducted to compare the effect of strengthening exercises on depression, fatigue and quality of life in both SLE and sedentary subjects. When comparing SLE and control sedentary subjects, it was discovered that SLE has more depression scales than control ( $73.92 \pm 0.6$ vs. $66.93 \pm 0.4$ in sedentary), but after exercising, SLE improved $18.3 \%$ versus $16.3 \%$ in sedentary, indicating highly significant differences between both groups. In addition, the fatigue severity scale is higher in SLE56.8 \pm 1.9 than control 41. \pm 13.2 , which may be explained by different comorbidities. Musculoskeletal complications account for $15 \%$ of all systems, followed by neuropsychiatric $11 \%$, cardiovascular 9\%, and hematologic 3\%, as well as osteoporosis, which is a potentially preventable complication of SLE. All of the preceding had a high mortality rate, so exercise is an essential part of SLE management to avoid these complications [9].

SLE patients also suffer from accelerated bone remodeling due to abnormalities in the immune system, such as an imbalance of inflammatory cytokines including interleukin-6, which accelerates bone remodeling [10]. Bultink et al. [11] reported that activity involved in weight-bearing can prevent bone remodeling. Kipen et al. [12] stated that regular exercise with a minimum of $40 \mathrm{~min}$ aerobic activity, four times per week is protective against bone mineral density loss, which commonly occurs among SLE patients, especially those on corticosteroid treatment. As a result, we choose 50 min of strengthening exercises three times per week.

Cumulative treatment by hydroxyl chloroquine was found to correlate with physical inactivity as reported by Margiotta et al., in their study [13]. Aside from the cardiovascular benefits conferred by antimalarial use in SLE patients, fatigue is associated with reduced exercise intensity and has been reported in up to $90 \%$ of patients treated with it [14]. Other factors that potentially contribute to fatigue are obesity, low physical activity, poor

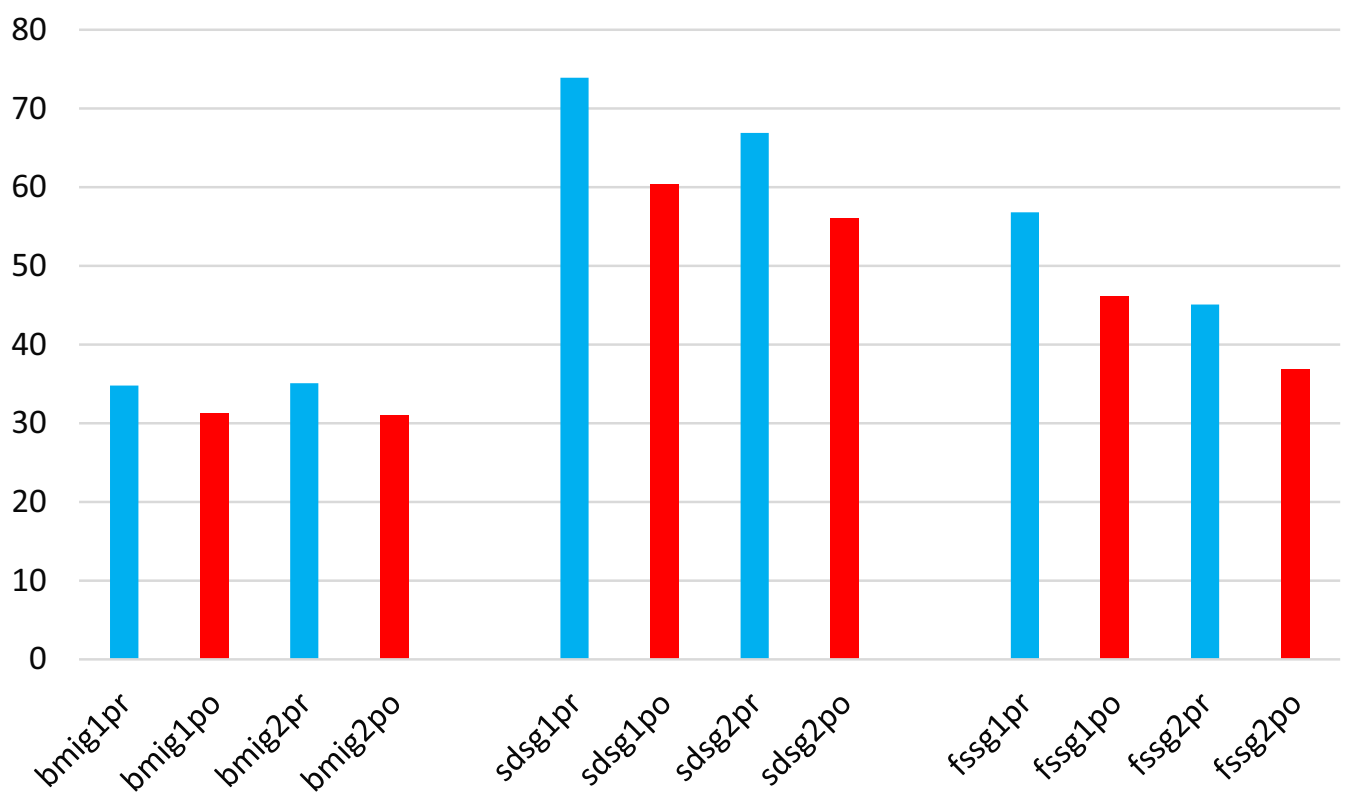

Fig. 1 Body mass index, severity of depression scale, fatigue severity scale 

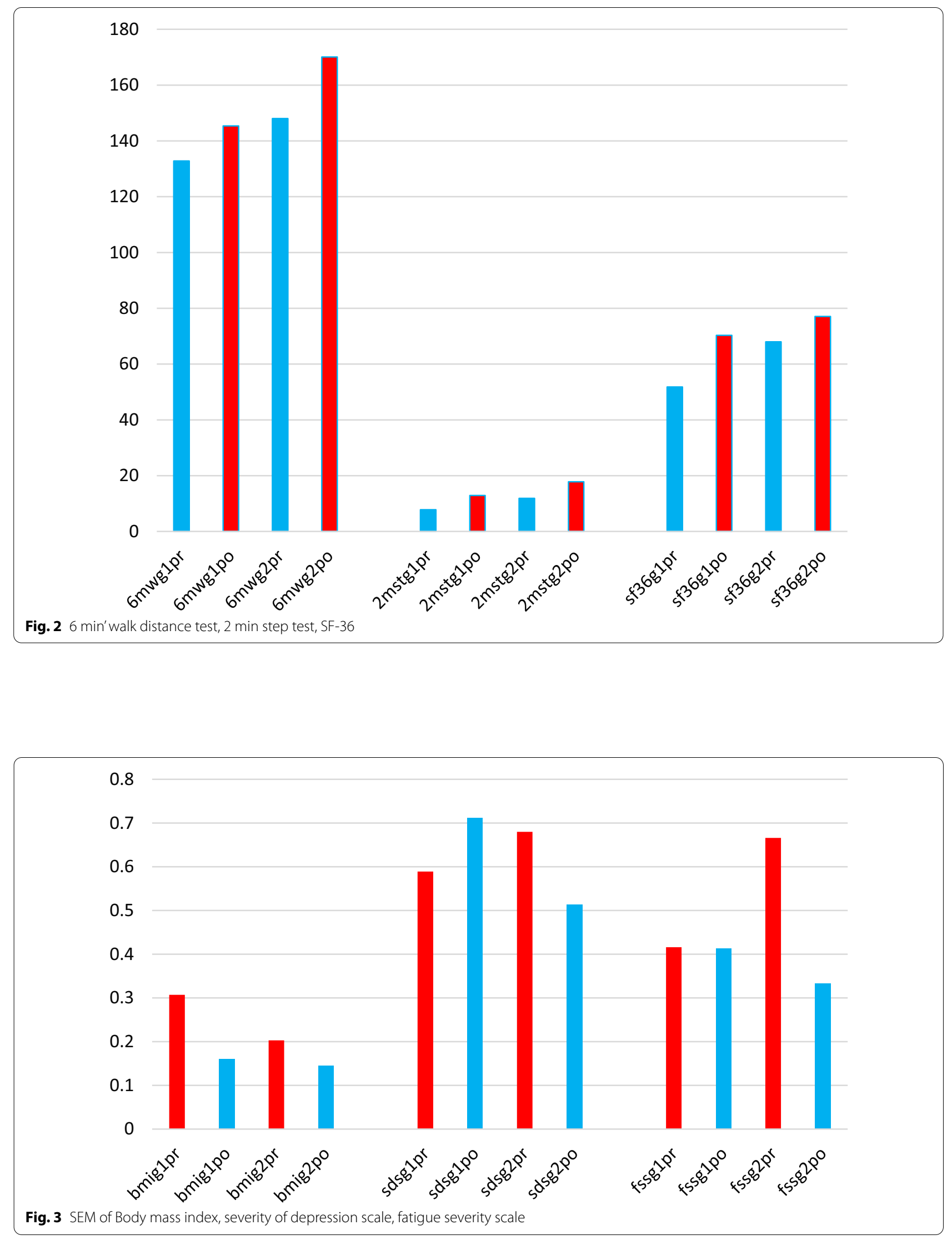


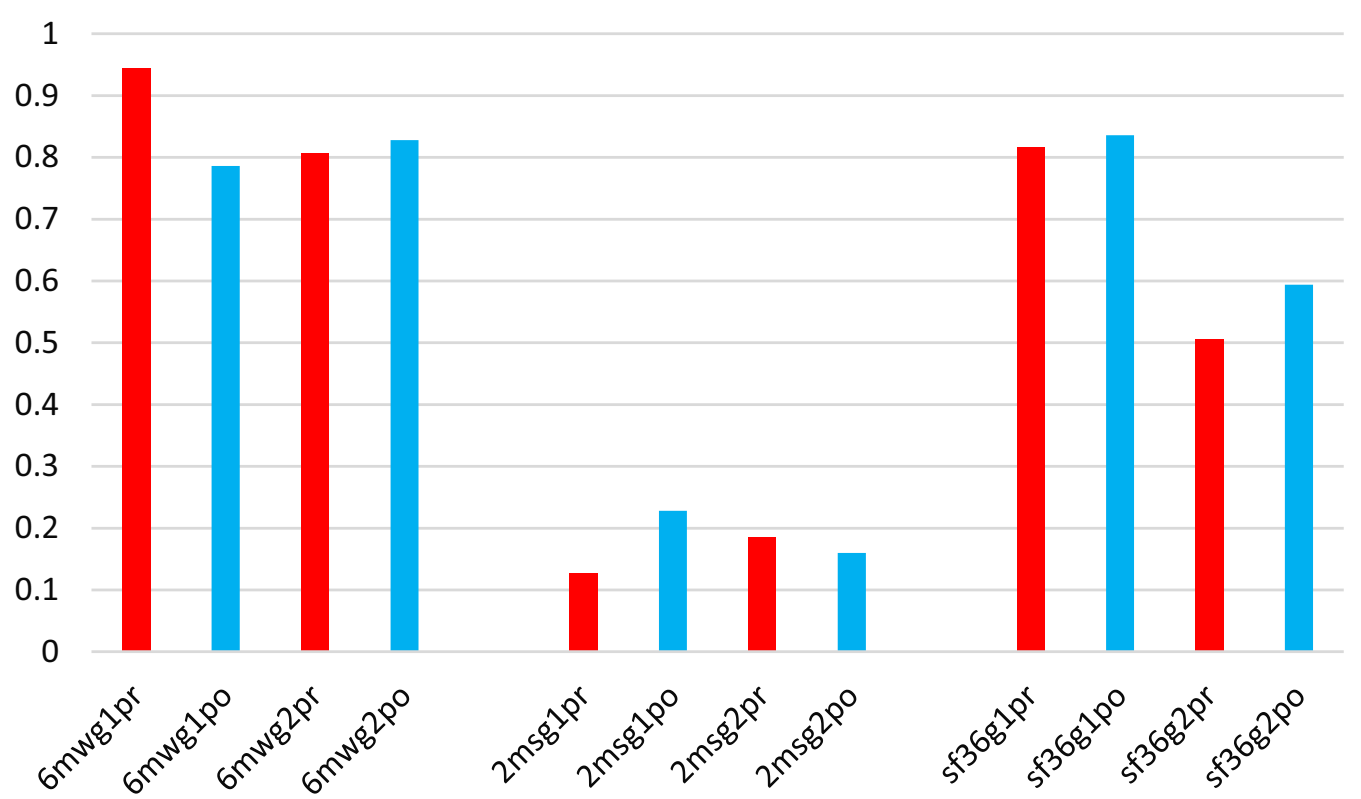

Fig. 4 SEM of 6-min walk distance test, 2-min step test, SF-36

Table 1 Pre and post measurements of SLE (group1)

\begin{tabular}{lccc}
\hline Item & Pretreatment group 1 & $\begin{array}{l}\text { Post } \\
\text { treatment } \\
\text { group 1 }\end{array}$ & P value \\
\hline BMI & $34.8 \pm 1.4$ & $31.3 \pm 0.7$ & $<0.001$ \\
SDS & $73.9 \pm 2.6$ & $60.4 \pm 3.2$ & $<0.001$ \\
FSS & $56.8 \pm 1.9$ & $46.1 \pm 1.8$ & $<0.001$ \\
6 min'walk test & $132.8 \pm 4.4$ & $145.4 \pm 3.6$ & $<0.001$ \\
2 min step test & $7.8 \pm .7$ & $12.9 \pm .1$ & $<0.001$ \\
SF-36 & $51.8 \pm 3.7$ & $70.3 \pm 3.7$ & $<0.001$ \\
\hline
\end{tabular}

Table 2 Pre and post measurements of control (group2)

\begin{tabular}{lccc}
\hline Item & $\begin{array}{l}\text { Pretreatment } \\
\text { group 2 }\end{array}$ & $\begin{array}{l}\text { Post treatment } \\
\text { group 2 }\end{array}$ & P value \\
\hline BMI & $35.1 \pm 1.0$ & $31.1 \pm 0.7$ & $<0.001$ \\
SDS & $66.9 \pm 3.4$ & $56 \pm 2.6$ & $<0.001$ \\
FSS & $45.1 \pm 3.2$ & $36.9 \pm 1.7$ & $<0.001$ \\
6 min'walk test & $148 \pm 4$ & $170.1 \pm 4.1$ & $<0.001$ \\
2 min step test & $11.9 \pm .9$ & $17.8 \pm 8$ & $<0.001$ \\
SF-36 & $68 \pm 2.5$ & $77.1 \pm 2.1$ & $<0.001$ \\
\hline
\end{tabular}

sleep quality, mood disorders, cognitive dysfunction, anxiety and vitamin D deficiency [15].

Nicholson et al. [16] compared the effect of $30 \mathrm{~min}$ of exercise, $5 \mathrm{~min}$ of warm-up, $5 \mathrm{~min}$ of cool-down, and 20 min of cycling on a bicycle ergometer three times per week for eight weeks to achieve $60-80 \%$ of their
Table 3 Percent of improvement of post treatment measures of both groups

\begin{tabular}{lll}
\hline Item & Group & $\begin{array}{l}\text { Percent of } \\
\text { improvement } \\
\text { (\%) }\end{array}$ \\
\hline BMI & Group1 & 10.05 \\
SDS & Group2 & 7.3 \\
& Group1 & 18.3 \\
FSS & Group2 & 16.3 \\
& Group1 & 18.8 \\
6 min'walk test & Group2 & 18.2 \\
& Group1 & 9.5 \\
2 min step test & Group2 & 14.9 \\
SF-36 & Group1 & 65.4 \\
& Group2 & 49.6 \\
& Group1 & 35.7 \\
\hline
\end{tabular}

maximum heart rate. Both groups are SLE patients divided randomly into both groups. There were significant differences in fatigue scale between both groups at the end of 8 weeks of exercise, $P \leq 0.005$, indicating significant improvement in fatigue in the exercise group.

The effects of two distinct exercise programs on chronic fatigue were examined in the Avaux et al. investigation [17]. Three groups of SLE patients were studied: supervised training, home training, and control. Patients were instructed to exercise on a bicycle for $3 \mathrm{~h}$ 
per week for 12 weeks until their heart rate reached at least $75 \%$ of their maximum heart rate, as well as strength workouts. The control group did not engage in any type of physical activity. There was a significant difference in the fatigue scale in both training groups after three months, but no significant difference in the fatigue score in the control group. Exercises can considerably reduce fatigue and depression [18].

In this study, SLE reported fatigue of $56.8 \pm 1.9$ pretreatment and $46.1 \pm 1.8$ post treatment versus $45.1 \pm 3.2$ pretreatment in sedentary subjects and $36.9 \pm 1.7$ post treatment. After three months of exercise, SLE improved to $18.8 \%$ versus $18.2 \%$ in sedentary subjects, and the number of cytokines secreted by skeletal muscle in response to muscle contraction can explain this. The first cytokine secreted is interleukin-6, interleukin 1 receptor antagonist, interleukin-10 and tumor necrosis factor receptor. Therefore, interleukin- 6 produced during physical exercise has been considered an anti-inflammatory rather than a pro-inflammatory cytokine [19].

During exercise, rapid activation of the sympathetic nervous system leads to much higher levels of catechol amines in circulation [20]. Patients with SLE report lower levels of vitality and general health than healthy controls, with SLE having a significant impact on physical functioning, psychological, emotional status, and social life [21].

When comparing SLE and sedentary control, aerobic capacity, which is measured by a 6 min walk test, it is decreased in SLE than in control. $132.8 \pm 4.4$ versus $148 \pm 4$, but after exercises, both groups had a highly significant difference between pre and post treatment, $145.4 \pm 3.6$ with $9.5 \%$ improvement in SLE versus $170.1 \pm 4.1$ with $14.9 \%$ improvement. Nicholson et al. [16] reported that SLE patients can improve their aerobic capacity (up to 19\%), and Tench et al. [22] reported that exercise tolerance and duration can be increased by $18 \%$ and $32 \%$ after performing aerobic exercise programs. In this study, aerobic capacity (6-min walk test) improved by $9.5 \%$ - and 2 -min step test improved by $65.4 \%$ in SLE compared to $14.9 \%$ and $49.6 \%$ in controls, respectively.

Ayan et al. [23] studied the effects of aerobic exercise on the rehabilitation of adults with SLE; it seems that the intensity, frequency and duration of the exercise program should be similar to those recommended in their population groups. In patients with low disease activity, moderate exercise proved to be safe, and different positive effects derived from engaging in it could be expected. These effects included the improvement of aerobic capacity, asthenia, tolerance to exercise, and possibly of physical function and depression. This explains why we choose the same exercises for both groups.
Physical exercise has been shown to have multiple benefits, including significant reduction in fatigue levels, weight loss, improvement in sleep quality, physical fitness and mood. QOL Meta-analysis of chronic fatigue syndrome research also showed that exercise reduced fatigue significantly after 12 weeks [24], and this backs up the findings of the current study, which found that BMI improved $10.05 \%$ and SF-36 improved $35.7 \%$ in SLE patients compared to $7.3 \%$ and $13.4 \%$ in controls. After treatment, there was a highly significant difference between the two groups, with SLE patients achieving greater improvement, which can be explained by greater improvement in fatigue severity score and depression in SLE patients.

Tench et al. [22] followed up on the fatigue severity of the participants 3 months after the exercise training and found that the participants who continued with regular exercise were more energetic than those who stopped exercising. Maintaining Exercise is important for physically inactive patients to extend the effects of training, and this may be due to the improvement in cardiovascular fitness, reduction of metabolic abnormalities, or fatigue, and consequently contribute to an improved QOL for individuals with SLE. The results of the present study finding agree with those reported by Ayan and Martin [25].

Carvalho et al. [26] showed a substantial increase in exercise tolerance, aerobic capacity, QOL, and depression symptoms after completing a supervised cardiovascular training program for individuals with SLE, which supports this concept. The findings of these two investigations are consistent with those of the current investigation. Physical performance, 6-min walk test, 2 min step test, SDS, and QOL all show significant variations.

Obesity is a prevalent concern in SLE, and obese patients are more likely to have a reduced functional capacity, a lower health-related quality of life, more fatigue and discomfort, and are more likely to develop hypertension and the metabolic syndrome [27].

In this study, there is a significant difference in BMI in both the SLE and control groups following exercises compared to baseline, which is in contrast to the findings of Tech et al. [22] who observed no significant difference in the three groups that participated in the study (exercises, relaxation, and control). Obesity and functional capacity have a negative relationship based on the difficulty of completing daily activities [28].

\section{Conclusions}

SLE has reduced exercise capacity due to fatigue, depression and drugs associated with several complications arising from disease progression. This leads to lower fitness levels, reduced exercise capacity, reduced muscle 
strength, greater disability, decreased quality of life and increased social dependence when compared to sedentary controls. Exercises can serve as helpful means of treatment that reduce fatigue, depression, improve exercise capacity, decrease weight and improve quality of life.

\section{Abbreviations}

SLE: Systemic lupus erythematosus; BMI: Body mass index; QOL: Quality of life; FSS: Fatigue severity score; SDS: Self-rating depression scale.

\section{Acknowledgements}

Not applicable.

\section{Authors' contributions}

MY design and implementation of the research, analysis of results and writing of the manuscript. The author read and approved the final manuscript.

\section{Funding}

Not applicable.

\section{Declarations}

\section{Ethics approval and consent to participate}

All procedures were carried out in compliance with the relevant committee on human experimentation's (institutional and national) ethical committee of faculty of physical therapy. Cairo University, as well as the Helsinki Declaration of 1975, as revised in 2000. All patients who agreed to participate in the study signed an informed consent.

\section{Consent for publication}

Not applicable.

\section{Availability of data materials}

The datasets used and/or analysed during the current study are available from the corresponding author on reasonable request.

\section{Competing interests}

The authors declare that they have no competing interests.

Received: 2 June 2021 Accepted: 11 December 2021

Published online: 20 December 2021

\section{References}

1. Somers EC, Marder W, Cagnoli P, Lewis E, Deguire P, Gordon C et al (2014) Population-based incidence and prevalence of systemic lupus erythematosus: the Michigan lupus epidemiology and surveillance program. Arthritis Rheumatol 66:369-378. https://doi.org/10.1002/art.38238

2. Estel GJ, Alarćon GS, Scofield L, Reinlib L, Cooper GS (2010) Understanding the epidemiology and progression of systemic lupus erythematosus. Semin Arthritis Rheum 39:257-268. https://doi.org/10.1016/j.semarthrit. 2008.10.007

3. Kiriakidou, M., Ching, C.A. Systemic lupus erythematosus. Ann Intern Med. 2013;159(7): Itc4-1. https://doi.org/10.7326/AITC202006020.

4. Di Battista M, Marcucci E, Elefante E, Tripoli A, Governato G, Zucchi D et al (2018) One year in review 2018: systemic lupus erythematosus. Clin Exp Rheumatol 36(5):763-777

5. Balsamo S, Neto L (2011) Fatigue in systemic lupus erythematosus: an association with reduced physical fitness. Autoimmun Rev 10:514-518. https://doi.org/10.1016/j.autrev.2011.03.005

6. O'Connor PJ, Puetz TW (2005) Chronic physical activity and feelings of energy and fatigue. Med Sci Sports Exerc 37(2):299-305. https://doi.org/ 10.1249/01.mss.0000152802.89770.cf

7. Tsokos, G.C. Systemic lupus erythematosus. N Engl J Med. 2011; 365: 2110-21. https://doi.org/10.1056/NEJMra1100359.
8. Ozel F, Argon G (2015) The effects of fatigue and pain on daily life activities in systemic lupus erythematosus. AĞRI J Turk Soc Algol 27(4):181189. https://doi.org/10.5505/agri2015.38278

9. Gordon, C. Long-term complications of systemic lupus erythematosus. Rheumatol (Oxford). 2002; 41(10):1095-1100. https://doi.org/10.1093/ rheumatology/41.10.1095.

10. Manolagas S, Jilka R (1995) Bone marrow, cytokines, and bone remodeling emerging insights into the pathophysiology of osteoporosis. N Engl J Med 332:305-311. https://doi.org/10.1056/NEJM199502023320506

11. Bultink I, Lems W, Kostense P, Dijkmans B, Voskuy A (2005) Prevalence of and risk factors for low bone mineral density and vertebral fractures in patients with systemic lupus erythematosus. Arthritis Rheum 54:20442050. https://doi.org/10.1002/art.21110

12. Kipen Y, Briganti E, Strauss B, Will R, Littlejohn G, Morand E (1999) Three year follow up of bone mineral density change in premenopausal women with systemic lupus erythematosus. J Rheumatol 26:310-317

13. Margiotta DPE, Basta F, Dolcini G, Batani V, Lo Vullo M, Vernuccio A et al (2018) Physical activity and sedentary behavior in patients with systemic lupus erythematosus. PLoS ONE 13:e0193728. https://doi.org/10.1371/ journal.pone.0193728

14. Arnaud L, Gavand PE, Voll R, Schwarting A, Maurier F, Blaison G et al (2019) Predictors of fatigue and severe fatigue in a large international cohort of patients with systemic lupus erythematosus and a systematic review of the literature. Rheumatology (Oxford) 58:987-996. https://doi. org/10.1093/rheumatology/key398

15. Ahn GE, Goldman R (2012) Fatigue in systemic lupus erythematosus. Int J Clin Rheumtol 7:217-227. https://doi.org/10.2217/JJR.12.4

16. Nicholson RL, Daltroy L, Eaton H, Gall V, Wright E, Hartley LH, Schur PH, Liang MH (1989) Effects of aerobic conditioning in lupus fatigue: a pilot study. Br J Rheumatol 28:500-505. https://doi.org/10.1093/rheumatolo gy/28.6.500

17. Avaux M, Hoellinger P, Husson SN, Fraselle V, Depresseux G, Frederic A (2016) Effects of two different exercise programs on chronic fatigue in lupus patients. Acta Clin Belg. https://doi.org/10.1080/17843286.2016. 1200824

18. Manal KY (2019) Effect of exercises training on fatigue, depression and physical activity in patients with systemic lupus erythematosus. J Health Med Res 1(1):4-11

19. Wu ML, Yu KH, Tsai JC (2017) The effectiveness of exercise in adults with systemic lupus erythematosus: a systematic review and meta-analysis to guide evidence- based practice. Worldviews Evid Based Nurs 14(4):306315. https://doi.org/10.1111/wvn.12221

20. Dimitrov S, Hulteng E, Hong S (2017) Inflammation and exercise: inhibition of monocytic intracellular TNF production by acute exercise via ß2-adrenergic activation. Brain Behav Immun 61:60-68. https://doi.org/ 10.1016/j.bbi.2016.12.017

21. Schmeding A, Schneider M (2013) Fatigue, health-related quality of life and other patient-reported outcomes in systemic lupus erythematosus. Best Prac Res Clin Rheumatol 27:363-375. https://doi.org/10.1016/j.berh. 2013.07.009

22. Tench C, McCarthy J, McCurdle I, White P, D'Cruz D (2003) Fatigue in systemic lupus erythematosus: a randomized controlled trial of exercise. Rheumatol (Oxford) 42:1050-1054. https://doi.org/10.1093/rheumatolo gy/keg289

23. Ayan C, de Munez P, Lemos M (2018) Effects of physical exercise in a population with systemic lupus erythematosus: a systematic review. Family Med Semergen 44(3):192-206. https://doi.org/10.1016/j.semerg.2017. 12.002

24. Neuberger, G.B., Press, A.N., Lindsley, H.B., Hinton, R., Cagle, P.E., Carlson. $K$, et al. Effects of exercise on fatigue, aerobic fitness, and disease activity measures in persons with rheumatoid arthritis. Res Nurs Health. 1997; 20(3):195-204. https://doi.org/10.1002/(sici 1098-240x (199706)20:3<195: aid-nur3>3.0.co; 2-d.

25. Ayan, C., Martin, V. Systemic lupus erythematosus and exercise. Lupus. 2007;16 (1):5-9. https://doi.org/10.1177/0961203306074795.

26. De Carvalho MR, Sato El, Tebexreni AS, Heidecher RT, Schenkman S, Neto TL (2005) Effects of supervised cardiovascular training program on exercise tolerance, aerobic capacity, and quality of life in patients with Systemic Lupus Erythematosus. Arthritis Care Res Off J Am Coll Rheumatol 53(6):838-844. https://doi.org/10.1002/art.21605 
27. Oeser A, Chung C, Asanuma Y, Avalos I, Stein M (2005) Obesity is an independent contributor to functional capacity and inflammation in systemic lupus erythromatosus. Arthritis Rheum 52:3651-3659

28. Chaiamnuay S, Bertoli AM, Fernandez M et al (2007) the impact of increased body mass index on systemic lupus erythromatosus: data from LUMINA, multiethnic cohort (LUMINA XLVI). J Clin Rhematol Prac Rep Musculoskeletal Dis 13(3):128-133

\section{Publisher's Note}

Springer Nature remains neutral with regard to jurisdictional claims in published maps and institutional affiliations.

\section{Submit your manuscript to a SpringerOpen ${ }^{\circ}$ journal and benefit from:}

- Convenient online submission

- Rigorous peer review

- Open access: articles freely available online

- High visibility within the field

- Retaining the copyright to your article

Submit your next manuscript at $\boldsymbol{\nabla}$ springeropen.com 